\title{
Tendencias del fitoplancton en el lago de la Albufera de Valencia e implicaciones para su ecología, gestión y recuperación
}

\author{
Susana Romo *, Ana García-Murcia, María José Villena, Vicent Sánchez y Alma Ballester
}

Área de Ecología, Facultad de Biología, Campus de Burjasot. Universitat de València. 46100-Burjasot. Valencia. España

* Corresponding author: Susana.Romo@uv.es

Recibido: 13/3/07

Aceptado: 30/7/07

\begin{abstract}
Phytoplankton trends in the lake of Albufera de Valencia and implications for its ecology, management, and recovery

The lake of Albufera de Valencia, with 2320 ha, is the largest shallow, oligohaline lake of the Spanish coast. It is located within the Natural Park of the Albufera de Valencia, which is mainly formed by rice fields. This important Spanish wetland is subjected to different environmental impacts. The Albufera Lake has been eutrophic since the 1970s. Even though a diversion plan for nutrients entering the lake mainly from the north and northwest started in 1991, its effects on the system's quality have been limited. Since 1985, a continuous monitoring of the lake's phytoplankton has been carried out, which represents one the largest temporal data set for shallow Mediterranean lakes. In this study, phytoplankton trends in the lake of the Albufera de Valencia are analysed, as well as its implications for the ecology, management, restoration, and conservation of the lake and its surrounding related wetlands. A $30 \%$ nutrient diversion has not decreased the dominance of cyanobacteria in the lake's phytoplankton, although it has contributed to a change in species composition, with a trend to a decrease in filamentous cyanobacteria and an increase of chroococcal cyanobacteria. The filamentous cyanobacteria Planktothrix agardhii, has been a good indicator species of the nutrient enrichment periods in the lake. The hydrological cycle of the lake was closely related to the phytoplankton ecology and the lake food webs. The reduction of the lake water inputs has produced higher algal biomasses and the increase of algal species with adaptation strategies towards more stable water columns. The increase of Microcystis aeuroginosa and Cylindrospermopsis raciborski in the lake implies an additional risk due to their capacity to produce cyanotoxins. Data analysis leads us to conclude that the reduction in the external nutrient loading was insufficient to revert the lake's trophic state towards an improvement of its water quality, which nowadays can be classified as "bad" according to the European Water Framework Directive. Some measures are suggested in order to manage and improve the water quality of the Albufera de Valencia lake.
\end{abstract}

Key words: Cyanobacteria, phytoplankton, shallow lake, Mediterranean, coastal.

\section{RESUMEN}

Tendencias del fitoplancton en el lago de la Albufera de Valencia e implicaciones para su ecología, gestión y recuperación

El lago de la Albufera de Valencia, con una extensión de 2320 ha, es el lago somero y oligohalino más extenso de nuestro litoral. Se encuentra englobado dentro del Parque Natural de la Albufera de Valencia, en su mayor parte formado por arrozales. Esta importante zona húmeda de España recibe diferentes impactos ambientales. El lago de la Albufera se encuentra en estado eutrófico desde los años 1970 hasta nuestros días y aunque en 1991 se inició un plan de desvío de los nutrientes que confluían en el lago, principalmente por su zona norte y noroeste, sus efectos sobre la calidad del sistema han sido limitados. Desde 1985 hasta el presente se ha realizado un seguimiento del fitoplancton del lago de la Albufera, lo que constituye una de las series temporales más largas que existen para los lagos someros de la cuenca mediterránea. En esta contribución se analizan las tendencias del fitoplancton en el lago de la Albufera de Valencia y sus implicaciones para la ecología, gestión, recuperación y conservación del mismo, y de sus zonas húmedas asociadas. El desvio de un $30 \%$ del aporte de nutrientes al lago no ha disminuido la dominante presencia de cianobacterias en su fitoplancton, aunque ha contribuido a un cambio en la composición de especies, con una tendencia de disminución de las cianobacterias filamentosas y un aumento de las cianobacterias croococales. La cianobacteria filamentosa Planktothrix agardhii ha sido una buena indicadora de los periodos de incrementos de nutrientes en el lago. El ciclo hidrologico del lago se relacionó estrechamente con la ecología del fitoplancton 
y la de sus redes tróficas. La reducción de la entrada de caudales al lago ha dado lugar a un aumento de la biomasa algal y de las especies algales con estrategias de adaptación a columnas de agua más estables. El aumento en el lago de Microcystis aeuroginosa y Cylindrospermopsis raciborski supone un riesgo añadido debido a su capacidad para producir cianotoxinas. El análisis de los datos nos hace concluir que la reducción en la carga externa de nutrientes fue insuficiente para revertir el estado trófico del lago hacia un estado de mejor calidad, que puede catalogarse de "malo" según la Directiva Marco del Agua. Se recomiendan una serie de medidas que permitan mejorar y gestionar el lago de la Albufera de Valencia.

Palabras clave: Cianobacterias, fitoplancton, lago somero, mediterráneo, costero.

\section{INTRODUCCIÓN}

El lago de la Albufera de Valencia, es el lago costero y oligohalino más extenso de la península Ibérica y se encuentra situado dentro del Parque Natural de la Albufera de Valencia. El Parque se caracteriza por su gran valor ecológico y está catalogado como zona húmeda de importancia internacional. Sin embargo, desde el siglo XIX, el lago de la Albufera sufre un fuerte impacto medioambiental, derivado de la actividad agrícola que se desarrolla en su marjal circundante y la presión demográfica e industrial en la zona. Todo ello, ha dado lugar a su progresiva degradación y hace que su gestión y conservación sea compleja dada la diversidad de actividades, usos e intereses que existen.

El lago de la Albufera de Valencia, al ser uno de los primeros lagos estudiados dentro de la historia de la limnología en España (Arévalo, 1916; 1917), posee una de las series históricas limnológicas más largas de nuestro territorio y de los países de la cuenca mediterránea (Zohary, 2004; Beklioglu et al., 2007). Estudios recientes muestran que existen marcadas diferencias en la ecología de los lagos someros dependiendo de sus zonas climáticas (Jeppesen et al., 2003; Moss et al., 2004; Romo et al., 2004; Jeppesen et al., 2005a y b; Beklioglu et al., 2007). El hecho de la existencia de una serie larga de datos en el lago de la Albufera de Valencia, sirve de referente para otros lagos someros mediterráneos, habida cuenta de la escasez de datos sobre la ecología de los mismos, respecto al conocimiento acumulado sobre los lagos someros de los países del norte y centro de Europa (Beklioglu et al., 2007). Por todo ello, esta base de datos limnológicos y del fitoplancton del lago de la Albufera resulta relevante e importante de mantener para la realización de estudios comparativos y predictivos (Anderson $e t$ $a l ., 2005$; Jeppesen et al, 2005a) y para el cumplimiento de las recomendaciones de la Directiva Marco del Agua (DMA) sobre conocimiento y seguimiento de los sistemas epicontinentales.

La Directiva Marco del Agua (Directiva 2000/60/CE), establece que todos los países de la Unión Europea deben prevenir el deterioro, mejorar y recuperar el estado de las masas de agua superficiales y potenciar que estén en buen estado ecológico. Asimismo, establece que se deben preservar las zonas protegidas, teniendo en cuenta los datos científicos y técnicos disponibles. Según esto, en el lago de la Albufera de Valencia, se debería recabar información sobre su estado de calidad y aplicar las medidas correctoras oportunas.

Actualmente, se está llevando a cabo en el lago de la Albufera un desvío parcial de la carga externa de nutrientes (Romo et al. 2005), dado que desde los años 1970 se encuentra en lo que se denomina una fase turbia según el modelo de estados alternativos de Scheffer et al. (1993). La disminución de la carga externa de nutrientes es un medida prioritaria en la recuperación de los lagos someros (Moss et al., 1996, Jeppesen et al., 2003), pero en ocasiones no resulta totalmente eficaz dada la reserva interna de nutrientes en el sedimento y su fácil resuspensión dentro de la columna de agua debido a la acción del viento o a la bioperturbación (Søndergaard et al., 2001). Así pues, frecuentemente es necesario llevar a cabo medidas complementarias, como por ejemplo, la biomanipulación de las redes tróficas, cuya finalidad es modificar las interacciones dentro de la red trófica, actuando sobre la intensidad de la depredación de los peces, el zooplancton, los ma- 
croinvertebrados y el flujo y reciclaje de los nutrientes (Moss et al., 1996).

La aproximación generalizada en el estudio del fitoplancton ha sido a través de los grupos taxonómicos, pero recientemente se ha propuesto una aproximación al estudio de la ecología del fitoplancton mediante grupos funcionales que comparten estrategias de adaptación a ciertas variables ambientales y biológicas (Reynolds et $a l .$, 2002). El diseño de los grupos funcionales fue originalmente descrito para los lagos templados del norte, pero algunos estudios confirman su validez y utilidad para el fitoplancton de los lagos someros de zonas tropicales y subtropicales (Huszar et al., 2000; Kruk et al., 2002), así como para los lagos someros mediterráneos (Romo \& Villena, 2005).

El presente trabajo tiene como objetivos estudiar la serie temporal de datos del fitoplancton del lago de la Albufera de Valencia desde 1985 y completarla con el periodo que abarca de 2001 a 2006. Con ello, se pretende obtener información científica sobre las tendencias y cambios del fitoplancton del lago y sobre su estado trófico, con el objetivo de que esta información ayude a definir y discutir las medidas complementarias a adoptar para su correcta recuperación y conservación, tal como demanda la Directiva Marco del Agua. Se estudiarán la biomasa, la abundancia, los grupos taxonómicos y funcionales del fitoplancton, así como su diversidad, tamaño y riqueza de especies. Especial hincapié se hará en el estudio de las tendencias observadas de estas variables y de los cambios tras el desvío de nutrientes al lago. Por último, los resultados obtenidos pretenden sumarse al sesgado conocimiento que se tienen de los lagos someros mediterráneos.

\section{MATERIAL Y MÉTODOS}

\section{Área de estudio}

La Albufera de Valencia es un lago somero y oligohalino (salinidad 1-2 \%o), situado en el Parque Natural de la Albufera $\left(210 \mathrm{~km}^{2}\right)$, cerca de la ciudad de Valencia $\left(39^{\circ} 20^{\prime} \mathrm{N}, 0^{\circ} 20^{\prime} \mathrm{W}\right)$. Su superficie es de 2320 ha y tiene una profundidad me- dia de $1.2 \mathrm{~m}$. La tasa media de renovación del agua se ha reducido de 10 a 7.4 año $^{-1}$ entre 1988 y 2004, debido a la menor entrada de caudales al sistema por cambios en la gestión del agua en su cuenca y por los periodos de sequía en años recientes (Soria \& Vicente, 2002; Soria, 2006). Existen marcadas diferencias estacionales de retención hidráulica en el lago en consonancia con el cultivo del arroz.

Aunque el cultivo del arroz en la zona tiene antecedentes anteriores, es desde finales del siglo XVIII cuando se intensifica su producción en las áreas circundantes del lago, tomando incluso la superficie misma del lago que se vio reducida en un $83 \%$ entre los siglos XVIII y XX (Rosselló, 1995). El lago funciona como un embalse de reserva de agua para los arrozales y el ciclo hidrológico del lago está supeditado al ciclo estacional del cultivo del arroz. El nivel de agua se controla mediante compuertas situadas en los tres canales de comunicación del lago con el mar. Durante enero-marzo las compuertas permanecen abiertas, permitiendo una alta tasa de renovación del agua del lago, para vaciar los campos antes de la siembra que se realiza en mayo, precedida de una fase de arado y fertilización. Entre mayo-septiembre, durante el crecimiento del arroz, el flujo de renovación del agua es mínimo y sólo aumenta después de la siega (septiembre-octubre). En noviembrediciembre, el nivel de agua del lago se incrementa para permitir el llenado de los campos en su máximo anual permaneciendo los campos inundados y sin cultivo (Romo \& Miracle 1993).

Los datos y estudios limnológicos muestran que aunque el lago tuvo diversos cambios de salinidad y usos a lo largo de su historia, el incremento exponencial de la población y de las industrias en la zona, especialmente a partir de los años 1960, produjo un cambio de estado trófico y degradación del lago que lo convirtió en un sistema hipertrófico (Dafauce, 1975; Rosselló, 1995; Romo et al., 2005). Hasta mediados del siglo XX, el lago de la Albufera de Valencia estaba densamente cubierto por macrófitos sumergidos y helófitos (p. e. Chara, Potamogeton, Myriophyllum y Ceratophyllum) (Arévalo, 1916; Pardo, 1942). El aumento de la eutrofización y 
el uso de pesticidas en el lago, provocó la desaparición de las plantas sumergidas y la dominancia del fitoplancton. La pérdida de vegetación sumergida afectó negativamente a la biodiversidad global del lago y a la estructura de sus redes tróficas (Dafauce, 1975; Romo et al., 2005). En la actualidad la vegetación sumergida se encuentra restringida a pequeñas zonas puntuales en las desembocaduras de ciertas acequias situadas principalmente de la zona sur, mientras la vegetación emergente se distribuye en la periferia y en el interior del lago formando sus islas o mates (Boira, 1987), aunque éstas se encuentran en un proceso de progresiva regresión (Argilés, 1997).

Respecto a la composición fitoplanctónica descrita para el lago de la Albufera, los trabajos de Pardo (1942) y Blanco (1974), constatan como al inicio de la década de los años 1970, las cianobacterias filamentosas de tener una presencia puntual pasan a dominar en el lago durante prácticamente la segunda mitad del año. Estudios más recientes del fitoplancton del lago, abarcan los periodos de 1980 a 1988 (Romo, 1991; Romo \& Miracle, 1993, 1994a y b, 1995; Romo, 1997) y de 1997 a 2000 (Villena \& Romo, 2003a y b), en los que se observa como el lago se mantiene en un estado eutrófico dominado por cianobacterias.

El zooplancton ha sido descrito en Blanco (1973, 1974), Alfonso \& Miracle (1990), Oltra \& Miracle (1992) y Miracle \& Sahuquillo (2002). Las interacciones tróficas en el lago de la Albufera, se describen en Romo et al (2005) y los cambios en las comunidades de peces durante el siglo XX en Blanco \& Romo (2006). Las características físico-químicas del lago de la Albufera de Valencia, han sido descritas en Serra et al. (1984), Miracle et al. (1987), Soria et al. (1987), Vicente \& Miracle (1992) y Soria et al. (2001).

El Plan de Saneamiento Integral del Parque Natural de la Albufera (Sánchez-Díaz, 1991) resolvió la construcción del Colector Oeste que recogería las aguas residuales que llegaban al Parque, principalmente procedentes de las zonas norte y oeste del lago, para su depuración en la Depuradora de Pinedo. Asimismo, estaba previsto que parte de las aguas residuales depuradas fuesen redirigidas de nuevo al lago. El Colector Oeste comenzó a funcionar parcialmente en
1991 y los sistemas de depuración de agua para las otras zonas circundantes del lago de la Albufera están todavía inacabados. En 1999, la carga externa total de fósforo que llegaba al lago se redujo en un $77 \%$ (de 18 a $4 \mathrm{~g} \mathrm{P} \mathrm{m}^{-2} \mathrm{año}^{-1}$ ) y la carga de nitrógeno en un $24 \%$ (de 324 a $247 \mathrm{~g} \mathrm{~N} \mathrm{~m}^{-2}$ año $^{-1}$ ) (Romo et al., 2005). La escasa reducción de la carga de nitrógeno se debe principalmente a que el lago continúa recibiendo la mayor parte de los aportes de aguas y cargas sin depurar procedentes de los arrozales de la zona sur y sureste del lago.

\section{Metodología}

La metodología seguida para el estudio del fitoplancton se mantuvo uniforme durante el periodo estudiado entre 1985-2006. Durante los años 1989-1994 no hay registro de datos y entre 19951997 sólo se tienen valores de clorofila-a como variable indicadora de la biomasa algal. Las muestras se recolectaron mensualmente en tres puntos de muestreo situados al norte, centro y sur del lago (Romo \& Miracle, 1993).

Las muestras de fitoplancton se tomaron en los primeros $50 \mathrm{~cm}$ de la columna de agua y fueron fijadas in situ con lugol en botellas de vidrio oscuras para su posterior estudio en el laboratorio. Estudios anteriores (Romo \& Miracle, 1993; Villena \& Romo, 2003b) y contrastes realizados para las muestras del periodo 2001-2006, no mostraron diferencias significativas en la composición del fitoplancton para los tres puntos muestreados (ANOVA, $p<0.05$ ), así pues el presente trabajo presenta el promedio entre los puntos de muestreo para cada una de las variables estudiadas. La clorofila-a del fitoplancton fue extraída de muestras sin fijar y analizada según APHA (1992), así como las concentraciones de fósforo total y los compuestos inorgánicos de nitrógeno (nitratos, nitritos y amonio). La temperatura, profundidad del disco de Secchi, $\mathrm{pH}$ y conductividad se midieron in situ.

Para la identificación y recuento del fitoplancton se utilizó un microscopio invertido y se siguió el método de Utermöhl (Utermöhl, 1958). Al menos se contaron 1000 individuos de la especie más abundante y 100 campos, con un error 
estimado de recuento del 4-6\% (Lund et al., 1958). El biovolumen algal fue estimado según aproximaciones geométricas (Rott, 1981; Hillebrand et al., 1999) y para ello se midieron entre 25 y 100 individuos (células o colonias) de cada especie. Se calculó el índice de diversidad de Shannon-Wiener y la equitatividad a partir de la abundancia de especies (Shannon \& Weaver, 1963). La riqueza de especies se calculó como el número de especies por muestra. Se determinó el GALD (mayor dimensión lineal o axial de una célula o colonia, Reynolds, 1984) separando las algas según presentaban GALD mayor o menor de $35 \mu \mathrm{m}$. Se seleccionaron como especies dominantes en el lago, aquellas que sumaron y representaron durante los periodos de estudio por- centajes de abundancia o biovolumen superiores o iguales al $80 \%$. Las especies dominantes se asignaron a un total de cinco grupos funcionales del fitoplancton de acuerdo con Reynolds et al. (2002). Así pues, el grupo funcional $S_{1}$ estuvo representado por Planktothrix agardhii, Pseudanabaena galeata, Planktolyngbya limnetica, Planktolyngbya contorta, Geitlerinema sp. y Geitlerinema amphibium; el grupo $S_{N}$ por Cylindrospermopsis raciborski; el grupo funcional $K$ por Aphanocapsa incerta y Aphanothece clathrata; el grupo M por Microcystis aeruginosa y por último el grupo $X_{1}$ por las especies Chroococcus dispersus y Chroococcus minutus.

La diferencia para cada una de las variables entre los periodos comparados de antes y des-
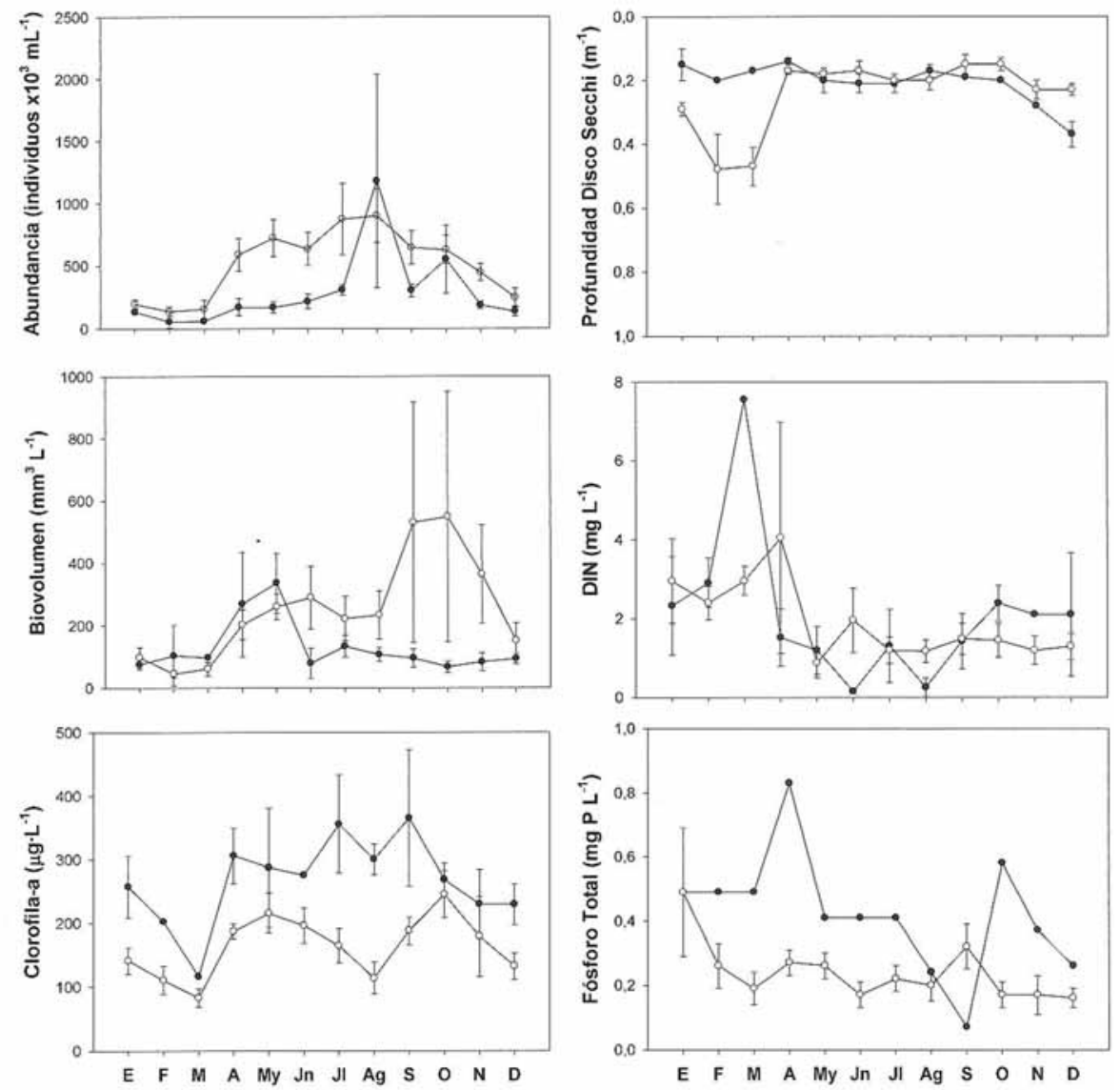

Figura 1. Cambios estacionales para varias variables en el lago de la Albufera durante el periodo 1985-1988 (círculos negros) y 1998-2006 (círculos blancos). Los valores representan medias mensuales junto con su error estándar. Seasonal changes for several variables in the Albufera of Valencia Lake during the period 1985-1988 (dark circles) and 1998-2006 (white circles). Values represent monthly means along with their standard errors. 
pués del desvío de nutrientes al lago (1985-1988 y 1998-2006) se analizaron mediante un test de Wilcoxon considerando las medias mensuales de cada variable obtenidas para cada periodo. El estudio de las tendencias de las principales especies del fitoplancton se realizó mediante medias agregadas y el método del ajuste analítico (Pérez, 2005). La correlación de Spearman se utilizó para estudiar las relaciones entre algunos pares de variables.

\section{RESULTADOS}

\section{Variables físico-químicas, clorofila-a, biovolumen y abundancia totales}

El periodo posterior al desvío de nutrientes conllevó una disminución significativa de la clorofila-a del fitoplancton y de la concentración de fósforo total en el lago (Tabla 1, Fig. 1). La fertilización estacional de los campos de arroz

Tabla 1. Variables limnológicas y del fitoplancton en el lago de la Albufera de Valencia para los periodos comparados de 1985-1988 y 1998-2006. El valor $p$ se refiere a un test de Wilcoxon para medias mensuales. Limnological and phytoplankton variables in the Albufera of Valencia Lake for the compared periods of 1985-1988 and 1998-2006. The p-value refers to a Wilcoxon's test for monthly means.

\begin{tabular}{|c|c|c|c|}
\hline & $1985-1988$ & 1998-2006 & $p$-valor \\
\hline Disco Secchi (m) & $0.21 \pm 0.02$ & $0.24 \pm 0.03$ & 0.97 \\
\hline Temperatura media anual $\left({ }^{\circ} \mathrm{C}\right)$ & $18.9 \pm 2.0$ & $19.4 \pm 1.9$ & 0.3 \\
\hline $\mathrm{pH}$ & $8.7 \pm 0.1$ & $8.9 \pm 0.1$ & 0.06 \\
\hline Conductividad $\left(\mu \mathrm{S} \mathrm{cm} \mathrm{cm}^{-1}\right)$ & $1832 \pm 102$ & $2046 \pm 71$ & 0.1 \\
\hline Fósforo total (mg P L $\left.{ }^{-1}\right)$ & $0.49 \pm 0.08$ & $0.23 \pm 0.02$ & 0.03 \\
\hline Nitratos $\left(\mathrm{mg} \mathrm{N} \mathrm{L}^{-1}\right)$ & $1.00 \pm 0.16$ & $1.65 \pm 0.3$ & 0.2 \\
\hline Amonio (mg N L $\left.{ }^{-1}\right)$ & $1.04 \pm 0.5$ & $0.56 \pm 0.07$ & 0.4 \\
\hline DIN (mg N L $\left.{ }^{-1}\right)$ & $2.1 \pm 0.6$ & $1.9 \pm 0.3$ & 0.7 \\
\hline Clorofila-a $\left(\mu \mathrm{g} \mathrm{L}^{-1}\right)$ & $266 \pm 20$ & $159 \pm 14$ & $<0.01$ \\
\hline Abundancia total (individuos $\times 10^{3} \mathrm{~mL}^{-1}$ ) & $289 \pm 90$ & $515 \pm 79$ & 0.012 \\
\hline Biovolumen total $\left(\mathrm{mm}^{3} \mathrm{~L}^{-1}\right)$ & $128 \pm 24$ & $251 \pm 47$ & 0.084 \\
\hline Biovolumen GALD $<35 \mu \mathrm{m}\left(\mathrm{mm}^{3} \mathrm{~L}^{-1}\right)$ & $15.7 \pm 2.5$ & $83,4 \pm 15.6$ & $<0.01$ \\
\hline Biovolumen GALD $>35 \mu \mathrm{m}\left(\mathrm{mm}^{3} \mathrm{~L}^{-1}\right)$ & $113 \pm 25$ & $167 \pm 41$ & 0.48 \\
\hline Diversidad (bits individuo $^{-1}$ ) & $2.5 \pm 0.12$ & $2.8 \pm 0.11$ & 0.02 \\
\hline Equitatividad ( \%) & $44 \pm 2.1$ & $52 \pm 2.0$ & $<0.01$ \\
\hline Riqueza especies ( $n^{\circ}$ especies por muestra) & $52 \pm 2.4$ & $43 \pm 1$ & 0.01 \\
\hline \multicolumn{4}{|l|}{ Biovolumen Fitoplancton $\left(\mathrm{mm}^{3} \mathrm{~L}^{-1}\right)$} \\
\hline Cianobacterias & $119 \pm 25$ & $228 \pm 48$ & 0.14 \\
\hline Cianobacterias filamentosas & $111 \pm 25$ & $60 \pm 13$ & $<0.01$ \\
\hline Cianobacterias croococales & $7.5 \pm 2.6$ & $168 \pm 49$ & $<0.01$ \\
\hline Cloroficeas & $2.5 \pm 0.2$ & $7.7 \pm 1.0$ & $<0.01$ \\
\hline Diatomeas & $5.4 \pm 0.7$ & $12.3 \pm 2.2$ & 0.01 \\
\hline Criptoficeas & $0.6 \pm 0.1$ & $1.6 \pm 0.2$ & $<0.01$ \\
\hline Euglenoficeas & $1.2 \pm 0.7$ & $0.2 \pm 0.05$ & 0.01 \\
\hline Dinoflagelados & $0.2 \pm 0.05$ & $0.5 \pm 0.1$ & $<0.01$ \\
\hline \multicolumn{4}{|l|}{ Especies Dominantes Biovolumen $\left(\mathrm{mm}^{3} \mathrm{~L}^{-1}\right)$} \\
\hline Aphanocapsa incerta & $1.35 \pm 0.5$ & $26.2 \pm 5.6$ & $<0.01$ \\
\hline Chroococcus dispersus & $0.03 \pm 0.02$ & $2.5 \pm 0.8$ & $<0.01$ \\
\hline Chroococcus minutus & $0.01 \pm 0.003$ & $1.0 \pm 0.3$ & $<0.01$ \\
\hline Cylindrospermopsis raciborskii & $0.8 \pm 0.4$ & $3.1 \pm 1.7$ & 0.01 \\
\hline Microcystis aeruginosa & $2.3 \pm 1.0$ & $121 \pm 43$ & $<0.01$ \\
\hline Planktolyngbya contorta & $0.25 \pm 0.13$ & $3.7 \pm 1.3$ & $<0.01$ \\
\hline Planktolyngbya limnetica & $2.13 \pm 1.5$ & $0.7 \pm 0.2$ & 0.43 \\
\hline Planktothrix agardhii & $96.2 \pm 26.8$ & $9.1 \pm 4.7$ & $<0.01$ \\
\hline Pseudanabaena galeata & $4.7 \pm 1.0$ & $38.6 \pm 9.7$ & $<0.01$ \\
\hline Cyclotella spp. & $3.3 \pm 0.6$ & $10.7 \pm 2.1$ & $<0.01$ \\
\hline
\end{tabular}


en abril-mayo y su vaciado durante septiembreoctubre implicaron un aumento de las entradas de nitrógeno y fósforo al lago en estas épocas (Fig. 1). La clorofila-a se correlacionó positivamente con el fósforo total en el periodo 19982006 ( $r=0.34, p<0.01)$ y negativamente con el nitrógeno inorgánico disuelto (DIN) $(r=-0.41, p<0.001)$. Las concentraciones de clorofila-a descendieron desde $266 \pm 20 \mu \mathrm{g} \mathrm{L}^{-1}$ de media anual hasta $159 \pm 14 \mu \mathrm{g} \mathrm{L}^{-1}$ entre 1985 y 2006 (Tabla 1, Fig. 2). Sin embargo, los valores tienden a estabilizarse en el periodo más reciente, y así para 2001-2006 la media fue de 145 $\pm 16 \mu \mathrm{g} \mathrm{L}^{-1}$ (máximo $366 \mu \mathrm{g} \mathrm{L}^{-1}$ en octubre de 2004 y mínimo $34 \mu \mathrm{g} \mathrm{L}^{-1}$ en febrero de 2006) (Fig. 2). Tras el desvío de nutrientes, se observaron varias fases claras en el lago con valores de clorofila-a por debajo de $10 \mu \mathrm{g} \mathrm{L}^{-1}$ (Fig. 2), de las cuales se estudiaron en detalle las de marzo de 1999 y febrero de 2000 (Romo et al., 2005). En febrero de 2006 se observó también una fase clara que afectó, sin embargo, sólo a la parte norte del lago. Estas fases claras se caracterizaron por un intenso efecto de depredación del zooplancton sobre el fitoplancton (mayoritariamente de Daphnia magna) y por valores de transparencia del disco de Secchi que llegaban hasta el fondo.

Estacionalmente la clorofila-a, se relacionó con el patrón estacional de apertura y cierre de las compuertas en el lago, con mínimos en los meses de febrero-marzo y los máximos entre abril y septiembre-octubre, coincidiendo el primero con un aumento del tiempo de renovación del agua tras del vaciado de los arrozales antes de la siembra y el segundo con la mayor estabilidad hidrológica durante el cultivo del arroz (Fig. 1).

A diferencia de la clorofila-a, la abundancia total del fitoplancton se incrementó significativamente entre los periodos comparados de 1985-1988 y 1998-2006 (Tabla 1, Fig. 1). Tal y como se observaba para las concentraciones de clorofila-a, la abundancia y el biovolumen totales del fitoplancton estuvieron interrelacionados con el ciclo hidrológico del lago, con máximos durante la segunda mitad del año coincidiendo con una mayor estabilidad hídrica y entrada de nutrientes al lago (Fig. 1).
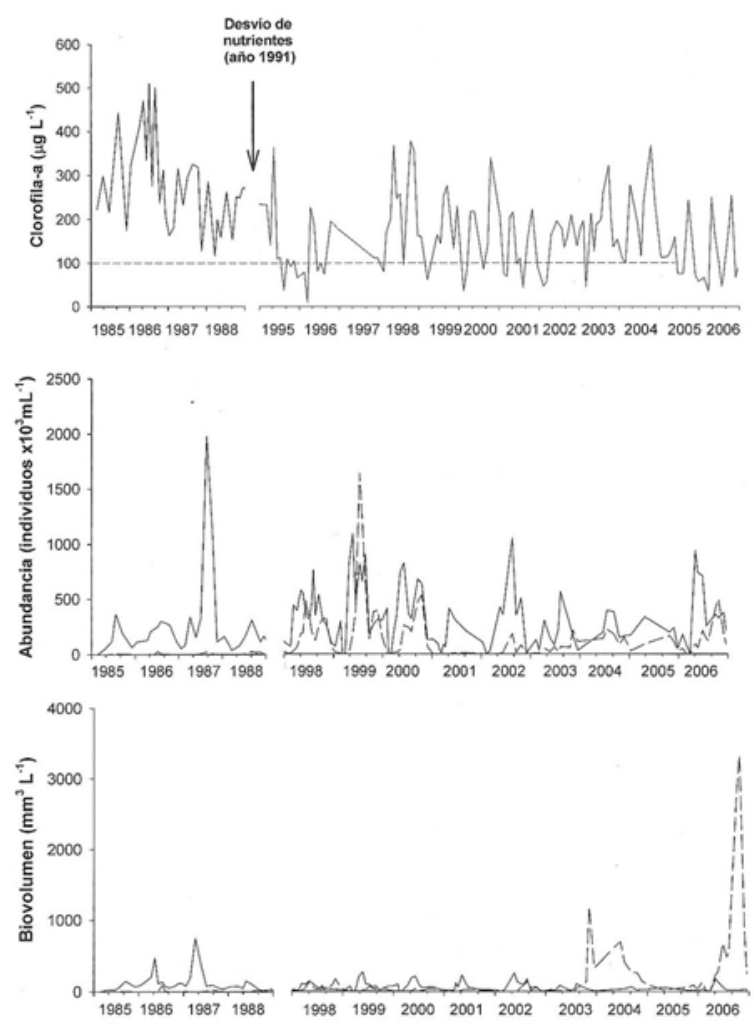

Figura 2. Cambios interanuales en la concentración total de clorofila-a del fitoplancton, abundancia y biovolumen de las cianobacterias filamentosas (línea continua) y croococales (línea discontinua) en el lago de la Albufera de Valencia entre 1985 y 2006. Interannual changes in the total concentration of phytoplankton's chlorophyll-a, abundance and biovolume of filamentous (dark line) and chrooococcal (dotted line) cyanobacteria in the Albufera of Valencia Lake between 1985 and 2006.

\section{Grupos taxonómicos y funcionales}

Las cianobacterias fueron el grupo dominante representando más del $76 \%$ de la abundancia y biovolumen totales del fitoplancton, durante el periodo 1980-2006 (Tabla 1). Sólo durante las fases claras se observó la sustitución de este grupo por la dominancia de pequeñas clorofíceas y diatomeas centrales. Se observa que las cianobacterias filamentosas dominaron principalmente durante el periodo de 1985-1988 (representaban $80 \%$ de la abundancia y biovolumen totales), mientras que para 1998-2006 existe un descenso significativo de este grupo (42\% biovolumen y $59 \%$ abundancia total) en favor principalmente de las cianobacterias croococales (Tabla 1, Fig. 2). 

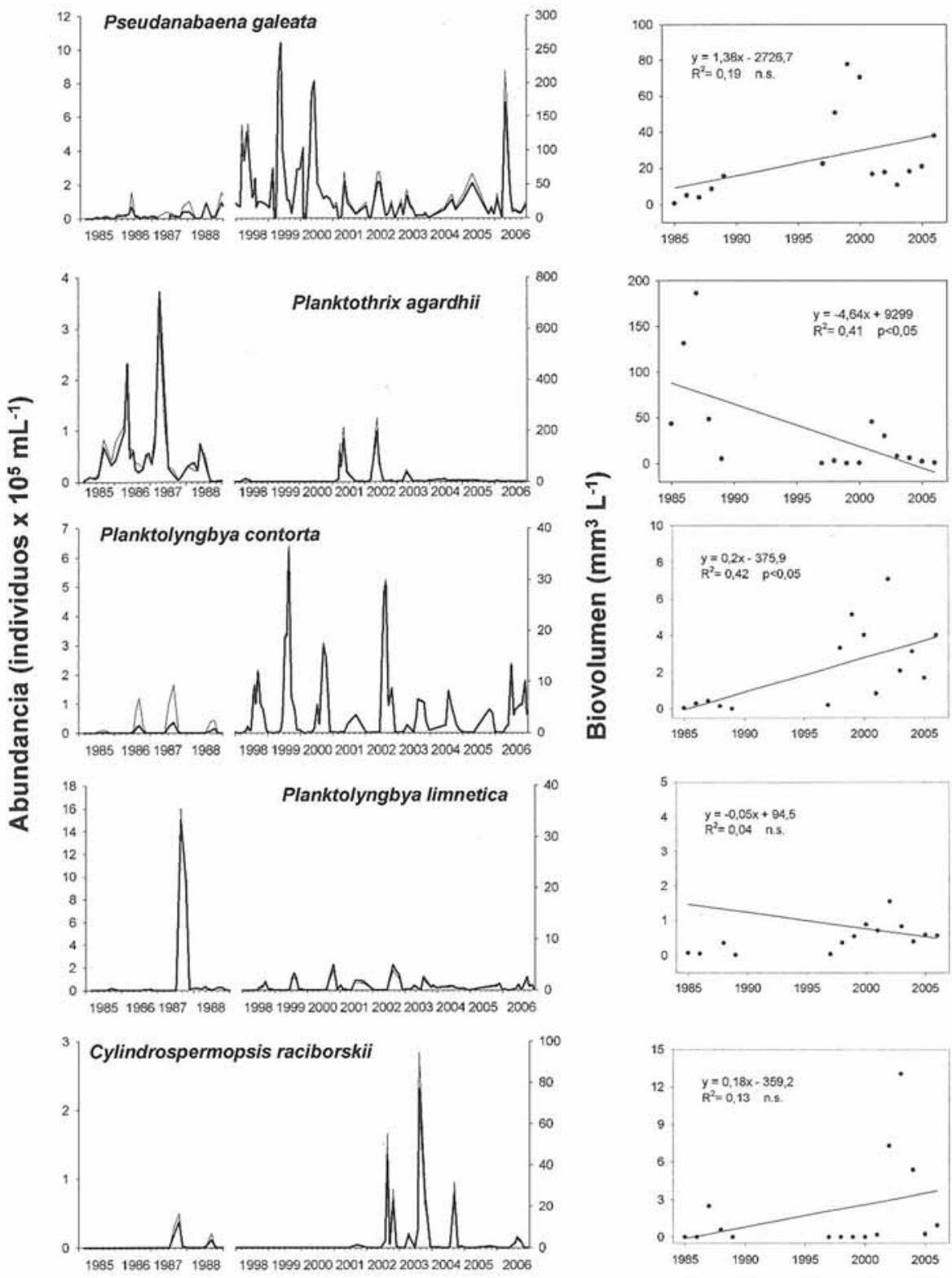

Figura 3. Cambios interanuales de la abundancia (línea fina) y biovolumen (línea gruesa) de las principales especies fitoplanctónicas en el lago de la Albufera de Valencia durante el periodo 1985-2006. En el margen derecho se representa la tendencia de cambio del biovolumen de cada una de las especies (n.s. = regresión lineal no significativa). Interannual changes in abundance (thin line) and biovolume (thick line) of the main phytoplankton species in the Albufera of Valencia Lake during 1985-2006. In the right margin, the change trend of the biovolume for each species is represented ( $n . s .=$ not significant linear regression).

La dinámica temporal de las principales especies se muestra en las figuras 3 y 4. Entre 1985 y 1988, Planktothrix agardhii representó el $67 \%$ de biovolumen medio anual en el lago. Sin embargo, estuvo prácticamente ausente en el perio- do posterior al desvío de nutrientes durante 19982000 y reapareció puntualmente durante 2001 y 2002 (Fig. 3). Durante 1998-2006, la oscilatorial dominante fue Pseudanabaena galeata contribuyendo para este periodo con un $34 \%$ a la 

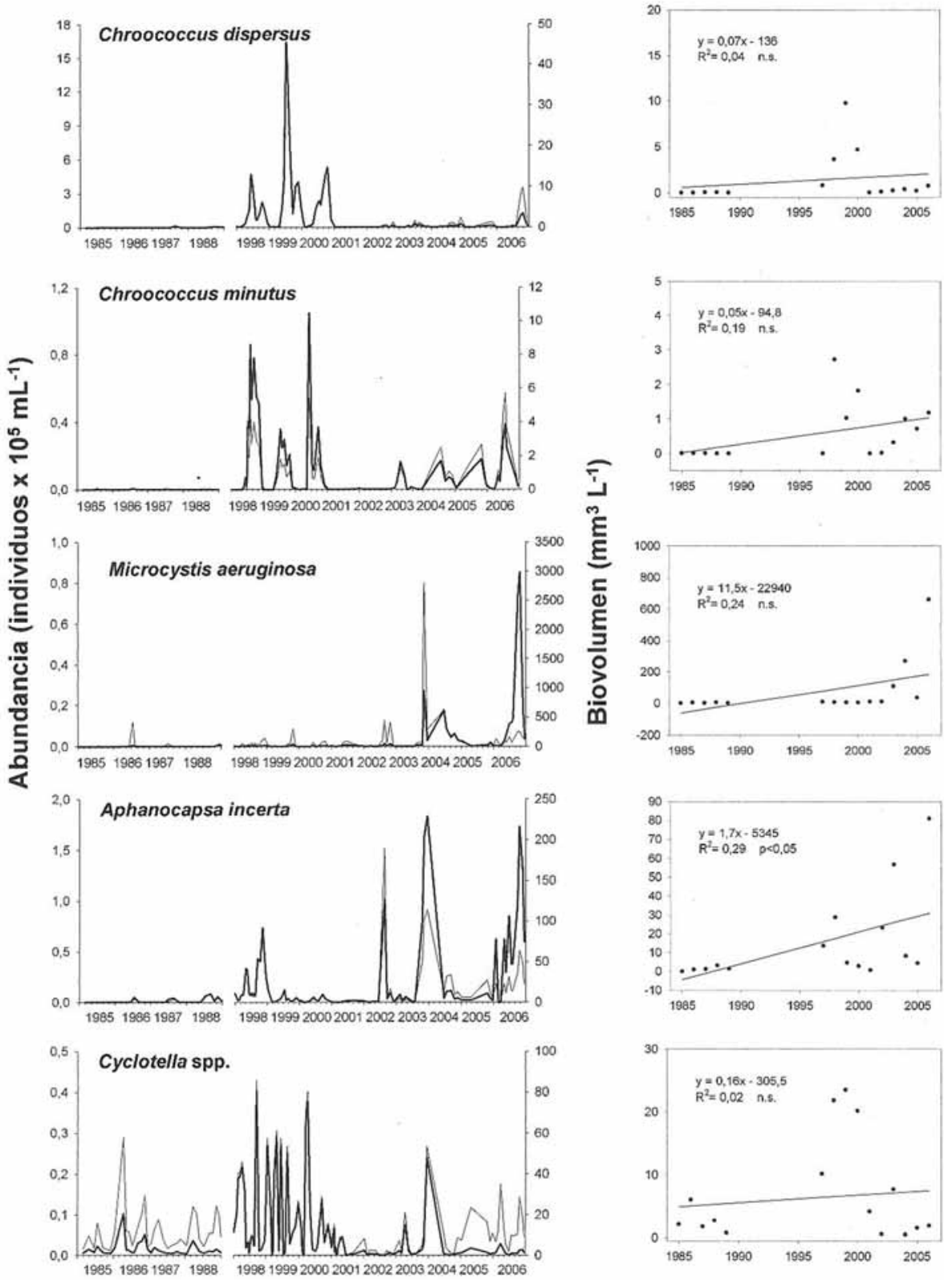

Figura 4. Cambios interanuales de la abundancia (línea fina) y biovolumen (línea gruesa) de las principales especies fitoplanctónicas en el lago de la Albufera de Valencia durante el periodo 1985-2006. En el margen derecho se representa la tendencia de cambio del biovolumen de cada una de las especies (n.s. = regresión lineal no significativa). Interannual changes in abundance (thin line) and biovolume (thick line) of the main phytoplankton species in the Albufera of Valencia Lake during 1985-2006. In the right margin, the change trend of the biovolume for each species is represented ( $n . s .=$ not significant linear regression).

abundancia media total del fitoplancton y con un $27 \%$ al biovolumen total. Otras cianobacterias filamentosas aumentaron también su presencia en el lago, como Planktolyngbya contorta y Cylin- drospermopsis raciborskii (Tabla 1, Fig. 3). Esta última especie, perteneciente al grupo de las nostocales, se incrementó especialmente a partir de 2002 (Fig. 3). Planktolyngbya limnetica no 


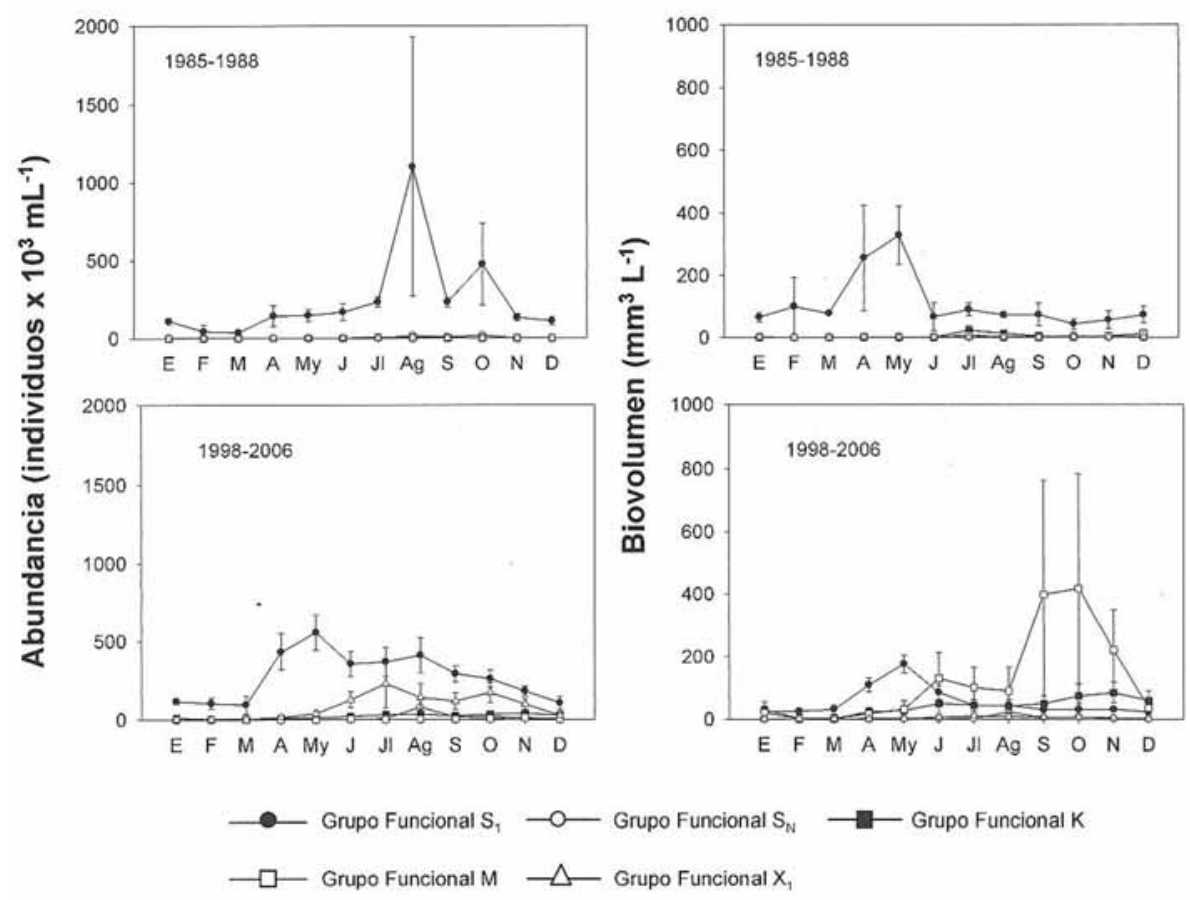

Figura 5. Cambios estacionales de abundancia y biovolumen de los principales grupos funcionales del fitoplancton en el lago de la Albufera de Valencia para los periodos comparados de 1985-1988 y 1998-2006. Los valores representan medias mensuales junto con su error estándar. Seasonal changes of abundance and biovolume of the main phytoplankton functional groups in the Albufera of Valencia Lake for the compared periods of 1985-1988 and 1998-2006. The values represent monthly means along with their standard errors.

mostró diferencias significativas en cuanto a su biovolumen medio en el lago entre los dos periodos estudiados (Tabla 1, Fig. 3).

Entre las cianobacterias croococales destacaron Chroococcus dispersus, que tuvo un patrón de distribución unimodal con máximos durante 1998-2000 (Fig. 4, Tabla 1). Esta especie, junto con Chroococcus minutus fueron las especies dominantes en verano durante 1998-2000. Sin embargo, a partir de 2002 se observa un cambio en la composición específica hacia la predominancia de croococales coloniales de gran tamaño como Microcystis aeruginosa y Aphanocapsa incerta (Fig. 4, Tabla 1). Estos cambios y substitución de especies quedó también reflejado en el GALD. Así pues, la dominancia de oscillatoriales con GALD > $35 \mu \mathrm{m}$ durante el periodo 1985-1988 dio paso a la de especies de menor tamaño (GALD $<35 \mu \mathrm{m}$ ) durante el periodo 1998-2000 y en los últimos años hacia un tamaño de algas y GALD grande (Tabla 1).
Así mismo, se observó en el fitoplancton del lago un cambio en la tendencia de los principales grupos funcionales y las estrategias adaptativas de las especies. Para el periodo 1985-1988, el grupo funcional dominante fue el grupo $\mathrm{S}_{1}$. Sin embargo, para 1998-2006 se observó además la presencia de otros grupos funcionales, como los grupos $\mathrm{X}_{1}, \mathrm{~S}_{N}, \mathrm{~K}$ y M (Fig. 5). Se constata pues, una tendencia hacia especies adaptadas a columnas de agua más estables o con escaso flujo de renovación del agua y estrategias adquisitivas respecto a los cambios en las variables ambientales (por ej., luz o nutrientes).

El resto de los grupos taxonómicos incrementaron su presencia en el lago tras el desvío de nutrientes, excepto las euglenofíceas (Tabla 1). Las clorofíceas fue el grupo taxonómico que presentó el mayor número de especies (96 de las 166 especies identificadas en el lago) y el segundo grupo en abundancia (1985-1988, media anual de $11 \pm 1 \cdot 10^{3}$ individuos $\mathrm{mL}^{-1}$ y para $1998-2006$ 
de $35 \pm 4 \cdot 10^{3}$ individuos $\mathrm{mL}^{-1}$ ). Durante 2005 y 2006 se observó un aumento de especies del género Chlamydomonas. Por otra parte, durante las fases claras de 1999 y 2000 se registró una dominancia de las clorofíceas Chlorella sp., Ankyra judayi y Chlamydomonas spp. y de las diatomeas centrales (Cyclotella spp.), mientras que durante la fase clara de 2006 sólo predominaron Chlamydomonas spp. y Cyclotella spp.

Las diatomeas no mostraron diferencias significativas en cuanto a su abundancia, pasando de una media anual de $13 \pm 2 \cdot 10^{3}$ individuos $\mathrm{mL}^{-1}$ en el periodo $1985-1988$ a $11 \pm 2 \cdot 10^{3}$ individuos $\mathrm{mL}^{-1}$ para 1998-2006. Sin embargo, las medias anuales de biovolumen casi se triplicaron (Tabla 1). La tendencia en la presencia de Cyclotella spp. fue unimodal, con una mayor abundancia y biovolumen entre 1998 y 2000 (Fig. 4).

Las criptofíceas, dinofíceas y euglenofíceas tuvieron una menor contribución cuantitativa en el fitoplancton del lago, con una presencia más esporádica y medias anuales de abundancia por debajo de $3 \cdot 10^{3}$ individuos $\mathrm{mL}^{-1}$. Las especies de criptofíceas del lago pertenecieron únicamente a los géneros Cryptomonas (4 especies) y Rhodomonas (2 especies). Las dinofíceas sólo estuvieron representadas por Gymnodinium cf. lacustre y Peridinium umbonatum. Las euglenofíceas presentaron nueve especies, destacando Euglena proxima y Phacus sp.

\section{Diversidad y riqueza de especies}

La diversidad de Shannon-Wiener y la equitatividad no mostraron una tendencia pronunciada de cambio para los periodos comparados de 1985-1988 y 1998-2006, aunque la riqueza de especies descendió desde 52 a 43 especies por muestra (Tabla 1). El rango de diversidad osciló entre 0,8 y 4,2 bits individuo ${ }^{-1}$. Estacionalmente, la diversidad mantuvo un patrón similar para la totalidad del estudio, con mínimos en abril-mayo, coincidiendo con los máximos principalmente de oscillatoriales (Figs. 2 y 6), seguido de un aumento progresivo y máximo a partir de septiembre (Fig. 6).

\section{DISCUSIÓN}

Diversos grupos algales, excepto las cianofíceas filamentosas y las euglenofíceas, aumentaron su contribución en el fitoplancton entre los periodos comparados antes y después del desvío de nutrientes. Todo ello indica un cambio en la composición específica del fitoplancton, en contraste con algunos estudios que postulan que una reducción en la carga de nutrientes debería afectar antes a la biomasa que a la estructura de la comunidad (Sas, 1989). Sin embargo, el lago de la Albufera de Valencia continúa dominado por cianobacterias después de la reducción de nutrientes llevada a cabo en 1991. La dominancia de las cianobacterias es común en los lagos someros de zonas cálidas (Reynolds, 1984; Osborne, 2005) y parece que pueden dominar bajo un amplio rango de nutrientes en los lagos someros de la zona mediterránea (Romo et al., 2004; Becklioglu et al., 2007). Pero aunque el lago continúa dominado por cianobacterias, existe una tendencia clara hacia la sustitución de las especies de oscilatoriales por croococales. Así pues, durante el periodo 1985-1988, el fitoplancton del lago estuvo dominado por tres especies de oscilatoriales (Planktothrix agardhii, Pseudanabaena galeata y Geitlerinema sp.) (Romo \& Miracle, 1993). Las oscillatoriales y concretamente Planktothrix agardhii, pueden formar monocultivos cuyas poblaciones limitan severamente la luz para el resto del fitoplancton (Scheffer et al, 1997; Scheffer, 1998). Planktothrix agardhii puede ser desplazada en el lago de la Albufera por otras oscilatoriales con tricomas más pequeños, bajo concentraciones menores de fósforo y mayor intensidad luminosa (Romo \& Miracle, 1993; Romo, 1994, Romo \& Miracle, 1994b). La reducción de la entrada de fósforo al lago probablemente influyó en su disminución y permitió a las poblaciones de otras especies de menor tamaño y con menores requerimientos de nutrientes que se desarrollasen en el lago, como por ej. Pseudanabaena galeata y Planktolyngbya contorta. Esta tendencia de aumento de las especies con menor tamaño ha sido también observada en otros lagos some- 

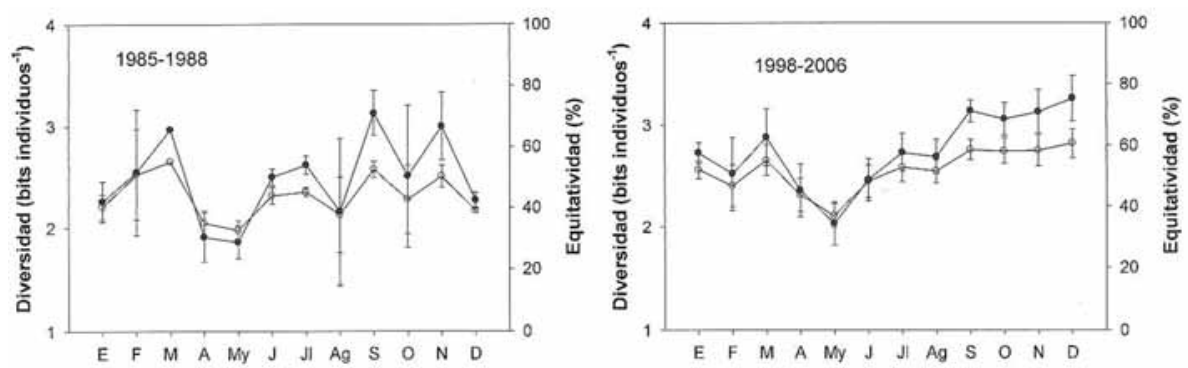

Figura 6. Cambios estacionales de diversidad (círculos negros) y equitatividad (círculos blancos) en el lago de la Albufera de Valencia para los periodos 1985-1988 y 1998-2006. Los valores representan medias mensuales junto con su error estándar. Seasonal changes in diversity (dark circles) and equitability (white circles) in the Albufera of Valencia Lake during the periods 1985-1988 and 1998-2006. The values represent monthly means along with their standard errors.

tidos a oligotrofización por reducción de nutrientes (Wojciechowski et al, 1988; Stoyneva, 1998; Köhler et al., 2000).

Existen evidencias experimentales de que las cianobacterias croococales del grupo funcional $\mathrm{X}_{1}$ (en el lago de la Albufera, Chroococcus dispersus y Chroococcus minutus) pueden sustituir a las oscilatoriales cuando la concentración de fósforo total es menor de $0.3 \mathrm{mg} \mathrm{L}^{-1}$, hay estabilidad de la columna de agua y una baja tasa de depredación por el zooplancton (Romo \& Villena, 2005). Estas condiciones se produjeron en el lago de la Albufera de Valencia principalmente en verano de 1998-2000, coincidiendo con los máximos de cianobacterias del grupo funcional $X_{1}$. Esta misma sustitución estacional entre oscilatoriales y croococales de pequeño tamaño ha sido observada en el Étang de Bolmon, una laguna somera, costera e hipertrófica al sur de Francia (Chomerat et al., 2005). El aumento y predominancia desde 2002 de las cianobacterias croococales de mayor tamaño y capacidad de flotación (Microcystis aeruginosa, Aphanocapsa incerta y en menor medida también Aphanothece clathrata) pudiera relacionarse con el aumento de los tiempos de retención hidráulica en el lago en los últimos años, debido a una menor entrada de caudales al Parque (Soria, 2006). El aumento de $M$. aeruginosa, junto con Cylindrospermopsis raciborskii, que son especies potencialmente productoras de cianotoxinas (Reynolds, 1997), evidencian un riesgo añadido al de su crecimiento masivo en el lago. Podríamos decir entonces, que tras la mejora observada en el lago después del desvío de nutrientes (1998-2000), se ha producido a posteriori un cambio de composición fitoplanctónica que parece reflejar un empeoramiento en su calidad como consecuencia de la escasa entrada de caudales. Aunque el grupo funcional dominante fue el grupo S1 de oscilatoriales, hay que destacar la mayor presencia de otros grupos como $\mathrm{M}$, $\mathrm{K}, \mathrm{S}_{N}$ y $\mathrm{X}_{1}$ (Fig. 5). Así pues, se observa en el lago un cambio desde una estrategia tipo $R$ de especies adaptadas a elevadas cantidades de nutrientes, polimixis y oligofotia, hacia una estrategia de tipo $S$ con algas adaptadas a columnas de agua estables o con escaso flujo de renovación del agua y una menor o variable disponibilidad de recursos (estrategias $R$ y $S$ según Reynolds, 1997).

Los resultados constatan, como en trabajos previos (Romo \& Miracle, 1993), que la dinámica y ecología del fitoplancton del lago de la Albufera de Valencia se encuentran estrechamente relacionadas con su ciclo hidrológico. Una mayor abundancia y biomasa algal se observó en los meses de mayor estabilidad hidrológica durante el cultivo del arroz y tras la fertilización de los campos (abril-octubre). Por el contrario, tiempos altos de renovación del agua en eneromarzo, propiciaron la disminución del fitoplancton y en algunos años la aparición en el lago de diversas fases claras. Según Scheffer et al. (2001) estos periodos de fases claras en los sistemas acuáticos, pueden dar lugar a cambios claves dentro del ecosistema y de sus redes tróficas que ayuden a su recuperación. La marcada estacionalidad, se observó también a nivel de la diversidad de especies. La diversidad y riqueza de 
especies aumentó durante la segunda mitad del año, en parte como consecuencia de la interrelación del lago con sus ecosistemas circundantes (arrozales y acequias), que durante el vaciado de los arrozales permite la entrada de especies alóctonas al lago (Romo, 1997). Los cambios fitoplanctónicos observados durante los periodos con mayores tiempos de renovación del agua en el lago apuntan a que la gestión del lago debería considerar el manejo de los tiempos de renovación del agua como una herramienta para su recuperación. Diversas experiencias en lagos someros holandeses con dimensiones similares al lago de la Albufera, ponen en evidencia su efectividad para acelerar la recuperación y el retorno de la vegetación sumergida y propiciar la posibilidad de aplicar medidas complementarias, como la biomanipulación de las comunidades de peces (Meijer \& Hosper, 1997). El lago necesitaría la entrada de un caudal de aproximadamente $150-200 \mathrm{hm}^{3}$ de agua de buena calidad (fósforo total menor a 0.1-0.05 $\mathrm{mg} \mathrm{L}^{-1}$, Romo et al., 2007; Soria, 2006) y no de aguas residuales, como primer paso para su recuperación.

El desvío de nutrientes en el lago de la Albufera de Valencia afectó sobretodo a la concentración de fósforo (Romo et al., 2005). Sin embargo, la carga externa de fósforo actual de aproximadamente $4 \mathrm{~g} \mathrm{P} \mathrm{m}^{-2}$ año $^{-1}$ (Romo et al., 2005), está todavía lejos de los valores recomendados para una posible reversión del estado turbio o para una recuperación de los macrófitos sumergidos en los lagos someros (Benndorf, 1990; Romo et al., 2007). Así mismo, se recomiendan cargas externas de fósforo de la mitad de la cantidad actual en el lago de la Albufera para iniciar proyectos de biomanipulación de la red trófica (Moss et al., 1996). En este sentido, se debería apoyar la actividad pesquera que tradicionalmente se lleva realizando en el lago y que actualmente está en regresión, dado que ayuda al control de las poblaciones de peces que limitan la presencia de macrozooplancton en el lago (Blanco et al., 2003; Blanco \& Romo, 2006). Otros autores (Moss, 2001; González Sagrario et al., 2005; James et al., 2005), además señalan que los valores de nitrógeno deben ser también bajos para propiciar el retorno o el mantenimiento de la vegetación sumergida $\left(<2 \mathrm{mg} \mathrm{TN} \mathrm{L}^{-1}\right)$. Las cargas externas de nitrógeno al lago se han reducido sólo un $24 \%$ (247 $\mathrm{g} \mathrm{N} \mathrm{m}^{-2}$ año ${ }^{-1}$, Romo et al., 2005) y los valores son altos y difíciles de rebajar debido a la actividad agrícola en la zona y a la lentitud de la puesta en marcha de las medidas para la depuración de las aguas en su cuenca. Al disminuir los aportes de fósforo en el lago, pero no los de nitrógeno, se ha producido un aumento del cociente DIN:TP que puede estar afectando también a la reducción de las cianobacterias filamentosas en el lago de la Albufera (correlación del cociente con el biovolumen de oscilatoriales para 1985-2006, $r=-0.27, p<0.05) \mathrm{y}$ que estaría en concordancia con lo observado en algunos estudios de re-oligotrofización de lagos someros (Jeppessen et al., 2005a).

\section{CONCLUSIONES}

A pesar de las actuaciones llevadas a cabo para la recuperación del lago de la Albufera mediante el desvío parcial de la carga externa de nutrientes, el lago sigue encontrándose en un estado de eutrofia que se puede calificar de malo de acuerdo con la DMA (Moss et al., 2003). El trabajo muestra que la composición de especies fue relevante para obtener información sobre su estado de calidad y apoya su uso como variable biológica discriminante, tal y como recomienda la DMA. Las medidas llevadas a cabo condujeron a una mejora puntual de su calidad con respecto a la década de los años 1980 y a la aparición más frecuente de fases claras en el lago, pero resultaron ser insuficientes para mejorar o revertir su estado actual. El lago de la Albufera de Valencia se encuentra en lo que se denomina una fase turbia según el modelo de estados alternativos (Scheffer et al., 1993), y para revertirla, entre otras medidas, es necesario disminuir aun más la carga de nutrientes que recibe hasta valores umbrales que permitan un cambio posible del sistema (fósforo total menor a 0.1-0.05 mg L ${ }^{-1}$, Moss et al., 1996, Jeppesen et al., 2003, Romo et al., 2004). Asimismo, es necesario aumentar los tiempos de renovación del agua en el lago con caudales próximos a los que se dan entre enero y marzo. El trabajo cons- 
tata que un aumento de los tiempos de retención del agua en el lago en los últimos años ha producido un cambio fitoplanctónico hacia especies de cianobacterias potencialmente tóxicas y productoras de crecimientos masivos especialmente difíciles de eliminar. La actual política de gestión del agua por parte de la administración, parece imponer unas condiciones hidrológicas al lago y a sus ecosistemas circundantes (Soria, 2006), que difícilmente podrán mejorar la calidad biológica del lago y mantener las zonas húmedas del Parque. Se recomienda además, apoyar y gestionar adecuadamente la actividad pesquera que tradicionalmente se lleva realizando en el lago desde antiguo, dado que controla las poblaciones de peces que influyen negativamente sobre la calidad del agua (Blanco et al., 2003; Blanco \& Romo, 2006). A pesar de ser la laguna oligohalina más extensa de nuestra península y estar catalogada como zona de especial protección nacional e internacional, los recursos que actualmente se destinan al estudio de su ecología o a su recuperación son escasos e insuficientes para cumplir lo estipulado por la DMA. Por otra parte, la serie histórica de datos limnológicos del lago de la Albufera demuestra que posee un valor indiscutible, para entender su ecología y llevar a cabo una gestión y recuperación adecuadas, así como para ayudar a la de otros lagos someros de la zona mediterránea, pero precisa de un reconocimiento y apoyo institucional.

\section{AGRADECIMIENTOS}

Agradecemos al Servicio de Calidad de Aguas de la Consellería de Territorio y Vivienda de la Generalitat Valenciana por facilitarnos los datos de física y química del lago durante el periodo 1998-2006. En especial a María Sauquillo, Felicidad Cuesta, Ruth García y José Tornero, así como a otros técnicos del Parque por su inestimable ayuda y colaboración durante los muestreos. A la Oficina Técnica Devesa-Albufera y especialmente a Juan Miguel Benavent y Salvador Ferrer por su especial colaboración durante los muestreos de algunos de los años estudiados. Agradecemos también la inestimable ayuda de D. Ga- briel Romo en la supervisión y diseño de parte del material de muestreo. Al Dr. Juan Miguel Soria por sus comentarios sobre la hidrología del lago de la Albufera de Valencia y por gentilmente facilitarnos los datos de física y química durante el periodo 1985-1988.

\section{BIBLIOGRAFÍA}

ALFONSO, M. T. y M. R. MIRACLE. 1990. Distribución espacial de las comunidades zooplanctónicas de la Albufera de Valencia. Scientia Gerundensis, 16(2): 11-25.

APHA 1992. Standard Methods for the Examination of Water and Wastewater, 18th edition. American Public Health Association. Washington D.C. $558 \mathrm{pp}$.

ANDERSON, N., E. JEPPESSEN \& M. SØNDERGAARD. 2005. Ecological effects of reduced nutrient loading (oligotrophication) on lakes: an introduction. Freshwat. Biol., 50: 1589-1593.

ARÉVALO, C. 1916. Indroducción al estudio de los Cladóceros del plancton de la Albufera de Valencia. Trabajo del Laboratorio de Hidrobiología Española (1). Anales del Instituto General y Técnico de Valencia, 1(1): 1-66.

ARÉVALO, C. 1917. Algunos rotíferos planctónicos de la Albufera de Valencia. Trabajo del Laboratorio de Hidrobiología Española (4). Anales del Instituto General y Técnico de Valencia, 2(8): 1-49.

ARGILÉS, R. 1997. Estudio de la evolución de las matas de la Albufera de Valencia. Propuesta para su restauración. UPV, ETSIA, $282 \mathrm{pp}$.

BEKLIOGLU, M., S. ROMO, I. KAGALOU, X. QUINTANA \& E. BÉCARES. 2007. State of the art in the functioning of shallow Mediterranean lakes: Workshop conclusions. Hydrobiologia, 584: 317-326.

BENNDORF, J. 1990. Conditions for effective biomanipulation: conclusions derived from whole-lake experiments in Europe. Hydrobiologia, 200/201: 187-203.

BLANCO, C. 1973. Estudio de los rotíferos de la Albufera de Valencia. Influencia de la contaminación sobre su distribución. Tesis de Licenciatura, Universidad de Madrid. $51 \mathrm{pp}$.

BLANCO, C. 1974. Estudio de la contaminación de la Albufera de Valencia y de los efectos de dicha contaminación sobre la fauna y flora del lago. 
Tesis Doctoral, Universitat de València (España), 193 pp.

BLANCO, S. y S. ROMO. 2006. Ictiofauna del lago de la Albufera de Valencia: evolución histórica y situación actual. Boletín de la Real Sociedad Española de Historia Natural. Sección Biología, 101(1-4): 45-56.

BLANCO, S., S. ROMO, M. J. VILLENA \& S. MARTÍNEZ. 2003. Fish communities and food web interactions in six shallow Mediterranean lakes. Hydrobiologia, 506-509: 473-480.

BOIRA, H. 1987. La vegetación de la Albufera de Valencia y sus bioindicadores. Fundación Universitaria San Pablo CEU. Valencia. 50 pp.

CHOMÉRAT, N., S. FAYOLLE \& A. CAZAUBON. 2005. Phytoplankton dynamics in a hypertrophic brackish lake in the South of France. Verh. Internat. Verein. Limnol., 29: 491-496.

DAFAUCE, C. 1975. La Albufera de Valencia. Un estudio piloto. Monografías ICONA, 4: 1-127.

GONZÁLEZ SAGRARIO, M. A., E. JEPPESEN, J. GOMÀ, M. SØNDERGAARD, J. P. JENSEN, T. LAURIDSEN \& F. LANDKILDEHUS. 2005. Does high nitrogen loading prevent clear-water conditions in shallow lakes at moderately high phosphorus concentrations? Freshwat. Biol., 50: 27-41.

HILLEBRAND, H., C. D. DÜRSELEN, D. KIRSCHTEL, U. POLLINGHER \& T. ZOHARY. 1999. Biovolume calculation for pelagic and benthic microalgae. J. Phycol., 35: 403-424.

HUSZAR, V. L. M., L. H. S. SILVA, M. MARINHO, P. DOMINGOS \& C. L. SANTANNA. 2000. Cyanoprokaryote assemblages in eight productive tropical Brazilian waters. Hydrobiologia, 424: 67-77.

JAMES C. S., J. FISHER, V. RUSSELL, S. COLLINGS \& B. MOSS. 2005. Nitrate availability and hydrophyte species richness in shallow lakes. Freshwat. Biol., 50: 1049-1063.

JEPPESEN, E., M. SØNDERGAARD, J. P. JENSEN $\&$ T. L. LAURIDSEN. 2003. Recovery from eutrophication: global perspectives. In: Freshwater Management- Global versus Local Perspectives. M. Kumagai \& W. F. Vincent (eds.): 135-152. Springer, Tokyo.

JEPPESEN, E., M. SØNDERGAARD, J. P. JENSEN, K. E. HAVENS, O. ANNEVILLE, L.CARVALHO, M. F. COVENEY, R. DENEKE, M. T. DOKULIL, B. FOY, D. GERDEAUX, S. E. HAMPTON, K. KANGUR, J. KÖHLER, S. HILT,
E. H. LAMMENS, T. L. LAURIDSEN, M. MANCA, M. R. MIRACLE, B. MOSS, P. NÕGES, G. PERSSON, G. PHILLIPS, R. PORTIELJE, S. ROMO, C. L. SCHELSKE, D. STRAILE, I. TATRAI, E. WILLÉN \& M. WINDER. 2005a. Lake responses to reduced nutrient loading - an analysis of contemporary long-term data from 35 case studies. Freshwat. Biol., 50: 1747-1771.

JEPPESEN, E., M. SØNDERGAARD, N. MAZZEO, M. MEERHOFF, C. C. BRANCO, V. HUSZAR \& F. SCASSO. 2005b. Lake restoration and biomanipulation in temperate lakes: relevance for subtropical and tropical lakes. In: Tropical Eutrophic Lakes: Their Restoration and Management. V. Reddy (ed.).: 331-349. Oxford \& IBH Publishing Co. Prt. Ltd., New Delhi.

KÖHLER, J., H. BEHRENDT \& S. HOEG. 2000. Long-term response of phytoplankton to reduced nutrient load in the flushed Lake Müggelsee (Spree system, Germany). Arch. Hydrobiol., 148: 209229.

KRUK, C., N. MAZZEO, G. LACEROT \& C. S. REYNOLDS. 2002. Classification schemes for phytoplankton: a local validation of a functional approach to the analysis of species temporal replacement. J. Plankton Res., 24: 901-912.

LUND, J. W. G., C. KIPLING \& E. D. LE CREN. 1958. The inverted microscope method of estimating algal numbers and the statistical basis of estimations by counting. Hydrobiologia, 11: 143-170.

MEIJER, M. L. \& H. HOSPER. 1997. Effects of biomanipulation in the large and shallow lake Wolderwijd, The Netherlands. Hydrobiologia, 342/343: 335-349.

MIRACLE, M. R., J. M. SORIA, E. VICENTE y S. ROMO. 1987. Relaciones entre la luz, los pigmentos fotosintéticos y el fitoplancton en la Albufera de Valencia, laguna litoral hipertrófica. Limnetica, 3: 25-34.

MIRACLE, M. R. \& M. SAHUQUILLO. 2002. Changes of life-history traits and size in Daphnia magna during a clear-water phase in a hypertrophic lagoon (Albufera of Valencia, Spain). Verh. Internat. Verein. Limnol., 28: 1203-1208.

MOSS, B., J. MADGEWICK \& G. PHILLIPS. 1996. A guide to the restoration of nutrient-enriched shallow lakes. Environmental Agency, Broads Authority, Manchester, $180 \mathrm{pp}$.

MOSS, B. 2001. The Broads. The People's Wetland. The New Naturalist. Harper Collins Publishers, London. 392 pp. 
MOSS, B., D. STEPHEN, C., ALVAREZ, E. BECARES, W. VAN DE BUND, S. E. COLLINGS, E. VAN DONK, E. DE EYTO, T. FELDMANN, C. FERNÁNDEZ-ALÁEZ, M. FERNÁNDEZALÁEZ, R. J. M. FRANKEN, F. GARCÍACRIADO, E. M. GROSS, M. GYLLSTRÖM, L.-A. HANSSON, K. IRVINE, A. JÄRVALT, J.-P. JENSEN, E. JEPPESEN, T. KAIRESALO, R. KORNIJOW, T. KRAUSE, H. KÜNNAP, A. LAAS, E. LILL, B. LORENS, H. LUUP, M. R. MIRACLE, P. NÕGES, T. NÕGES, M. NYKÄNEN, I. OTT, W.PECZULA, E. T. H. M. PEETERS, G. PHILLIPS, S. ROMO, V. RUSSELL, J. SALUJÕE, M. SCHEFFER, K. SIEWERTSEN, H. SMAL, C. TESCH, H. TIMM, L. TUVIKENE, I. TONNO, T. VIRRO, E. VICENTE $\&$ D. WILSON. 2003. The determination of ecological status in shallow lakes-a tested system (ECOFRAME) for implementation of the European Water Framework Directive. Aquatic Conserv. Mar. Freshwat. Ecosyst., 13: 507-549.

MOSS, B., D. STEPHEN, D. BALAYLA, E. BÉCARES, S. E. COLLINGS, C. FERNÁNDEZALÁEZ, M. FERNÁNDEZ-ALÁEZ，C. FERRIOL, P. GARCÍA, J. GOMÁ, M. GYLLSTRÖM, L.-A. HANSSON, J. HIETALA, T. KAIRESALO, M. R. MIRACLE, S. ROMO, J. RUEDA, V. RUSSELL, A. STAHL-DELBANCO, M SVENSSON, K VAKKILAIEN, M VALENTÍN, W. J. VAN DE BUND, E. VAN DONK, E. VICENTE \& M. J. VILLENA. 2004. Continental-scale patterns of nutrient and fish effects on shallow lakes: synthesis of a pan-European mesocosm experiment. Freshwat. Biol., 49: 1633-1649.

OLTRA, R. \& M. R. MIRACLE. 1992. Seasonal succession of zooplankton populations in the hypertrophic lagoon Albufera of Valencia (Spain). Arch. Hydrobiol., 124: 187-204.

OSBORNE, P. 2005. Eutrophication of shallow tropical lakes. In: The lakes handbook. P. O'Sullivan \& C. S. Reynolds (eds): 278-299. Blackwell Science, Oxford.

PARDO, L. 1942. La Albufera de Valencia. Biología de las aguas continentales II. Instituto Forestal de Investigaciones y Experiencias, Madrid, 263 pp.

PÉREZ, C. 2005. Técnicas estadísticas con SPSS 12. Aplicaciones al análisis de datos. Prentice Hall. 802 pp.

REYNOLDS, C. S. 1984. The ecology of Freshwater Phytoplankton. Cambridge University Press, Cambridge. $384 \mathrm{pp}$.
REYNOLDS, C. S. 1997. Vegetation process in the pelagic: a model for ecosystem theory. Excellence in Ecology. Kinne, O. (ed.). ECI, Oldendorf. $371 \mathrm{pp}$.

REYNOLDS, C. S., V. L. HUSZAR, C. KRUK, L. NASELLI-FLORES \& S. MELO. 2002. Towards a functional classification of the freshwater phytoplankton. J. Plankton Res., 24: 417-428.

ROMO, S. 1991. Estudio del fitoplancton de la Albufera de Valencia, una laguna hipertrófica y somera, entre 1980 y 1988. Tesis Doctoral, Universidad de Valencia. 197 pp.

ROMO, S. 1994. Growth parameters of Pseudanabaena galeata Böcher in culture under different light and temperature conditions. Arch. Hydrobiol. Algolog. Stud., 75: 239-248.

ROMO, S. 1997. Importance of allochthonous phytoplankton in a coastal freshwater lake. Verh. Internat. Verein. Limnol., 26: 610-614.

ROMO, S. \& M. R. MIRACLE. 1993. Long-term periodicity of Planktothrix agardhii, Pseudanabaena galeata and Geitlerinema sp. in a shallow hypertrophic lagoon, the Albufera of Valencia (Spain). Arch. Hydrobiol., 126: 469-486.

ROMO, S. \& M. R. MIRACLE. 1994a. Long-term phytoplankton changes in a shallow hypertrophic lake, Albufera of Valencia (Spain). Hydrobiologia, 275-276: 153-164.

ROMO, S. \& M. R. MIRACLE. 1994b. Population dynamics and ecology of subdominant phytoplankton species in a shallow hypertrophic lake (Albufera of Valencia, Spain). Hydrobiologia, 273: 37-56.

ROMO, S. \& M. R. MIRACLE. 1995. Diversity of phytoplankton assemblages from a polimictic hypertrophic lake. Arch. Hydrobiol., 132: 363-384.

ROMO, S., M. R. MIRACLE, M. J. VILLENA, J. RUEDA, C. FERRIOL \& E. VICENTE. 2004. Mesocosm experiments on nutrient and fish effects on shallow lake food webs in a Mediterranean climate. Freshwat. Biol., 49: 1593-1607.

ROMO, S. \& M. J. VILLENA. 2005. Phytoplankton strategies and diversity under different nutrient levels and planktivorous fish densities in a shallow Mediterranean lake. J. Plankton. Res., 27: 12731286.

ROMO, S., M. J. VILLENA, M. SAHUQUILLO, J. M., SORIA, M. GIMÉNEZ, T. ALFONSO, E. VICENTE \& M. R. MIRACLE. 2005. Response of a shallow Mediterranean lake to nutrient diversion: 
does it follow similar patterns as in northern shallow lakes? Freshwat. Biol., 50: 1706-1717.

ROMO, S., M. J. VILLENA \& A. GARCÍAMURCIA. 2007. Epiphyton, phytoplankton and macrophyte ecology in a shallow lake under in situ experimental conditions. Fund. \& Appl. Limnol. Arch. Hydrobiol., 170.

ROSSELLÓ, V. M. 1995. L'Albufera de València. Publicacions de l'Abadia de Montserrat, Barcelona. $190 \mathrm{pp}$.

ROTT, E. 1981. Some results from phytoplankton counting intercalibrations. Schweiz. Z. Hydrol., 43: 34-62.

SÁNCHEZ DÍAZ, J. 1991. Plan Especial de Protección del Parque Natural de la Albufera. Generalitat Valenciana. Conselleria de Medi Ambient. Valencia. $148 \mathrm{pp}$.

SAS, H. 1989. Lake restoration by reduction of nutrient loading: expectations, experiences, extrapolations. Academia Verlag Richarz, Sankt Augustin, 497 pp.

SCHEFFER, M. 1998. Ecology of Shallow Lakes. Chapman and Hall, London. 357 pp.

SCHEFFER, M., S. H. HOSPER, M. L. MEIJER, B. MOSS \& E. JEPPESEN, 1993. Alternative equilibria in shallow lakes. Trends Ecol. Evol., 8: 275279.

SCHEFFER, M., S. RINALDI, A. GRAGNANI, L. R. MUR \& E. H. VAN NES. 1997. On the dominance of filamentous cyanobacteria in shallow, turbid lakes. Ecology, 78: 272-282.

SCHEFFER, M., D. STRAILE, E. H. VAN NES \& H. HOSPER. 2001. Climatic warming causes regime shifts in lake food webs. Limnol. Oceanogr., 46: $1780-1783$.

SHANNON, C. E. \& W. WEAVER. 1963. The Mathematical Theory of Communication. University of Illinois Press, Urbana, Illinois, $117 \mathrm{pp.}$

SERRA, M., M. R. MIRACLE y E. VICENTE. 1984. Interrelaciones entre los principales parámetros limnológicos de la Albufera de Valencia. Limnetica, 1: 9-19.

SØNDERGAARD, M., J. P. JENSEN \& E. JEPPESEN. 2001. Retention and internal loading of phosphorus in shallow, eutrophic lakes. The Scientific World, 1: 427-442.

SORIA, J. M., M. R. MIRACLE y E. VICENTE. 1987. Aporte de nutrientes y eutrofización de la Albufera de Valencia. Limnetica, 3: 227-242.

SORIA, J. M., E. VICENTE \& M. R. MIRACLE. 2001. The influence of flash floods on the limnology of the Albufera of Valencia lagoon (Spain). Verh. int. Ver. Limnol., 27: 2232-2235.

SORIA, J. M. y E. VICENTE. 2002. Estudio de los aportes hídricos al parque natural de la Albufera de Valencia. Limnetica, 21: 105-115.

SORIA, J. M. 2006. Past, present and future of la Albufera of Valencia Natural Park. Limnetica, 25: 135-142.

STOYNEVA, M. P. 1998. Development of the phytoplankton of the shallow Srebarna Lake (northeastern Bulgaria) across a trophic gradient. Hydrobiologia, 369-370: 259-267.

UTERMÖHL, H. 1958. Zur vervollkommung der quantitativen phytoplankton-methodik. Mitt. Int. Ver. Limnol., 9: 1-38.

VICENTE, E. \& M. R. MIRACLE. 1992. The coastal lagoon Albufera de Valencia: an ecosystems under stress. Limnetica, 8: 87-100.

VILLENA, M. J. \& S. ROMO. 2003a. Temporal changes of cyanobacteria in the largest coastal Spanish Lake. Arch. Hydrobiol. (Suppl.) (Algol. Stud.), 109: 593-608.

VILLENA, M. J. \& S. ROMO. 2003b. Phytoplankton changes in a shallow Mediterranean lake (Albufera of Valencia, Spain) after sewage diversion. Hydrobiologia, 506-509: 281-287.

WOJCIECHOWSKI, I., W. WOJCIECHOWSKA, K. CZERNAS, J. GALEK \& K. RELIGA. 1988. Changes in phytoplankton over a ten-year period in a lake undergoing de-eutrophication due to surrounding peat bogs. Arch. Hydrobiol., 78: 373-387.

ZOHARY, T. 2004. Changes to the phytoplankton assemblage of lake Kinneret after decades of a predictable, repetitive pattern. Freshwat. Biol., 49: 1355-1371. 